\section{6}

${ }^{1}$ Department of Physiology, Institute for Cardiovascular Research, VU University Medical Center, Amsterdam, The Netherlands

${ }^{2}$ Department of Pulmonary Diseases, Institute for Cardiovascular Research, VU University Medical Center, Amsterdam, The Netherlands

\section{Correspondence to}

Dr Anton Vonk Noordegraaf Department of Pulmonary

Diseases, VU University Medical Center, De Boelelaan 1117. Amsterdam 1081 HV The Netherlands; a.vonk@ vumc.nl

Published Online First 20 June 2016

\section{SLinked}

http://dx.doi.org/10.1136 thoraxjnl-2015-207949

CrossMark

To cite: Aman J Bogaard HJ, Vonk Noordegraaf A. Thorax 2016:71:767-769.

\title{
Why vessels do matter in pulmonary disease
}

\author{
Jurjan Aman, ${ }^{1,2}$ Harm Jan Bogaard, ${ }^{2}$ Anton Vonk Noordegraaf ${ }^{2}$
}

In addition to structural abnormalities of the diaphragm, congenital diaphragmatic hernia (CDH) presents with severe hypoplasia of lung tissue involving both the pulmonary vasculature and the airways. These developmental defects often yield a severe phenotype of pulmonary hypertension $(\mathrm{PH})$ and lung function impairment. Despite adequate surgical repair of the diaphragm, neonatal mortality of $\mathrm{CDH}$ remains high, due to ineffectiveness of inhaled nitric oxide (NO) and absence of alternative therapy for $\mathrm{PH}$ in the newborn.

Russo et $a l^{1}$ demonstrate that maternally administered sildenafil reaches therapeutic levels in the rabbit fetus, without toxicity for mother or the fetus. $\mathrm{CDH}$ fetuses treated with sildenafil demonstrated increased pulmonary vascular branching compared with placebo-treated fetuses. The improved vascularisation was paralleled by reduced pulmonary artery pressure and by morphological changes in the lung parenchyma and functional improvement. In line with previous rodent studies, Russo et $a l^{1}$ found that the decreased lung weight due to $\mathrm{CDH}$ is not rescued in sildenafil-treated fetuses with $\mathrm{CDH}$, suggesting that the sildenafil-induced improvement of vascular and alveolar development contributes to the lung maturation more than the volume growth. These wellperformed studies obtained in an animal model that closely resembles both the pharmacokinetics of human pregnancy and alveolar development in humans provide a next step towards a clinically useful therapy for $\mathrm{CDH}$ and its postnatal consequences.

In addition to providing relevant steps towards clinical development of sildenafil as a potential therapy for $\mathrm{CDH}$, Russo et al give valuable insights into the mechanisms of lung development. Stressing the key role of cyclic guanosine monophosphate (cGMP)/NO signalling in $\mathrm{CDH}$ pathophysiology, the study provides additional support for the idea that appropriate vascularisation critically determines final structure and function of the lung. ${ }^{2}$ The first mechanism suggested here is the involvement of vascular endothelial growth factor (VEGF), as the sildenafil-induced improvement in vascularisation and alveolar maturation was paralleled by an increase in VEGF expression in parenchymal cells. VEGF is of paramount importance in lung development, ${ }^{3}{ }^{4}$ as it contributes to pulmonary angiogenesis and vessel growth, with subsequent alveolar maturation and adequate matching of ventilation and perfusion. ${ }^{3}$ During normal lung development, VEGF is secreted by lung mesenchymal and alveolar epithelial cells, creating an parenchymal VEGF gradient ${ }^{5}$ that coordinates bronchovascular growth (figure 1). The decreased VEGF expression in the lung parenchyma, as described by Russo et al, is a consistent finding in $\mathrm{CDH}$, both in humans ${ }^{6}$ and in animal models. ${ }^{7}$ Reduced VEGF directly impairs lung development, as a number of studies have shown that decreased VEGF expression per se (eg, by heterozygous knockout of the hypoxia-inducible factor (HIF)-2a or by pharmacological VEGF-receptor inhibitors like SU-5416) is sufficient to induce the phenotype of hypovascularisation, alveolar immaturity and PH. ${ }^{8}{ }^{9}$ This is further supported by observations in bronchopulmonary dysplasia, a lung developmental disorder that shares many features with $\mathrm{CDH}$, including impaired VEGF expression, decreased vascular branching and impaired alveolar maturation. ${ }^{10}{ }^{11}$ In bronchopulmonary dysplasia, VEGF gene therapy reversed the phenotype of decreased vascular branching and alveolar maturation ${ }^{12}$ and could be mimicked by sildenafil treatment. ${ }^{11}$

As sildenafil was primarily developed to mimic the action of $\mathrm{NO}$, a second mechanism involved here may be the NO/cGMP signalling. Although not directly addressed in this paper, earlier rodent studies demonstrated that $\mathrm{CDH}$ pups have increased phosphodiesterase type 5 (PDE5) activity, paralleled by reduced cGMP and endothelial NO synthase (eNOS) concentrations. ${ }^{7}$ Like VEGF, lung vascular development is dependent on intact $\mathrm{NO} /$ cGMP signalling. Cigarette smoke studies have shown the critical dependence of endothelial cells on eNOS phosphorylation and NO secretion for endothelial tube formation and vasculogenesis, which could be rescued by NO donors. ${ }^{13}$ In line with these in vitro data, animal studies demonstrated that cigarette smoke-induced impairment of NO signalling results in $\mathrm{PH}$ and loss of alveoli. ${ }^{14}$ Of interest here, cigarette smoke-exposed mice and guinea pigs show pulmonary vascular remodelling before alveolar loss occurs, thereby stressing the dependence of mature lung parenchyma on vascularisation. This suggests that vascular changes may even underlie overt airway diseases in adults like emphysema/COPD, ${ }^{15}$ thus supporting recent insights that emphysema is "...characterized by a failure in cell and molecular maintenance programs necessary for proper lung development". ${ }^{16}$ Thus, VEGF-dependent and NO/cGMP-dependent vascular growth and maintenance are key determinants of lung function.

An important issue that remains to be addressed is why $\mathrm{CDH}$ leads to decreased VEGF. Although data from bronchopulmonary hypoplasia models point toward dysregulation of HIF-1a and HIF-2a signalling, ${ }^{17}$ no mechanistic studies have been performed to answer this question in $\mathrm{CDH}$. Future studies focusing on this question may yield valuable insights in gene regulation programmes in 
Figure 1 Illustration showing vascular endothelial growth factor (VEGF)-driven angiogenesis in normal lung development and congenital diaphragmatic hernia (CDH). (A) VEGF is secreted by lung parenchyma and the tip of growing airways in which VEGF production may be regulated by cyclic guanosine monophosphate (cGMP)-mediated hypoxia-inducible factor (HIF) activation. VEGF-driven angiogenesis matches perfusion to ventilation, thus creating a mature alveolocapillary unit. (B) In CDH, increased phosphodiesterase type 5 (PDE5) activity suppresses VEGF expression, which can be treated with sildenafil.

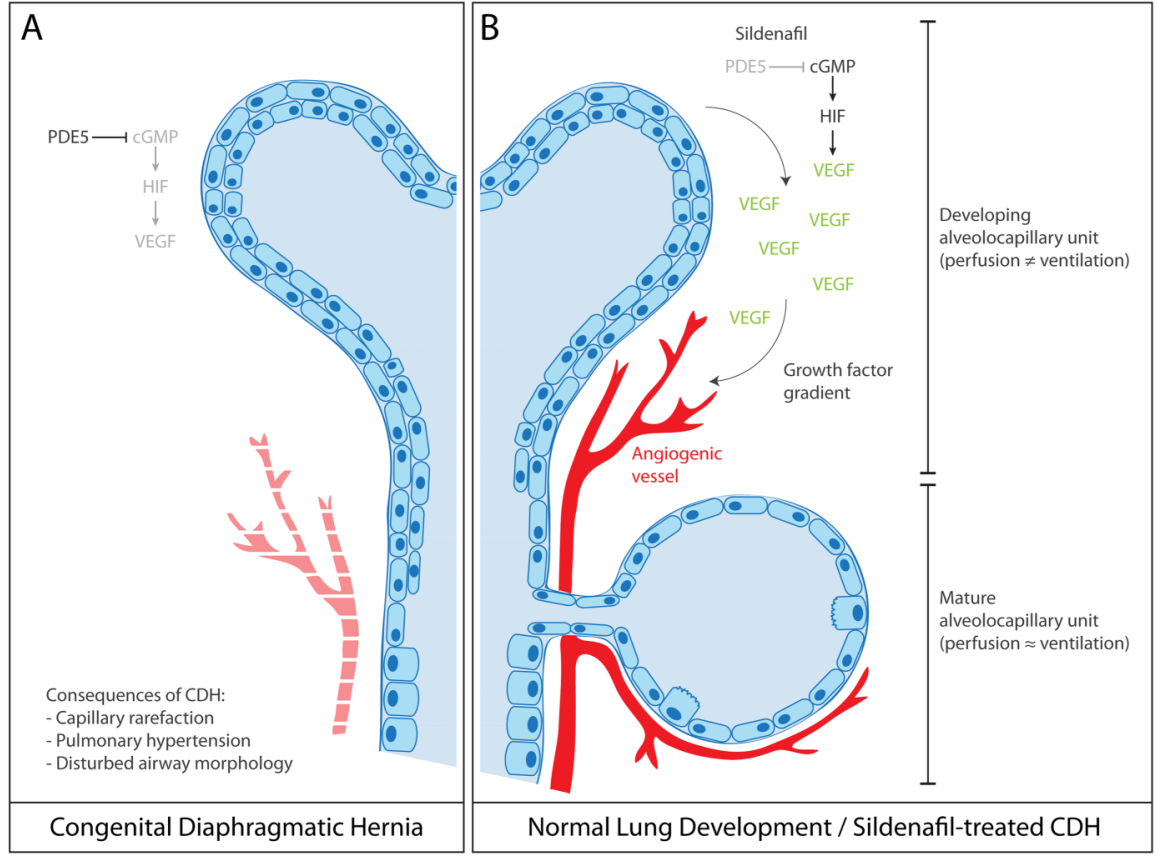

pulmonary development. A second question is whether and how VEGF and NO/cGMP signalling are linked. The observation that expression levels of VEGF and eNOS/cGMP are both decreased in $\mathrm{CDH}$ and are both enhanced by sildenafil ${ }^{7}$ suggests that VEGF and eNOS/cGMP share a common pathway. In the classical pathway, VEGF stimulates eNOS, which leads to NO release which may in turn stimulate cGMP. This pathway, however, does not explain why sildenafil enhances VEGF expression, as VEGF acts upstream of cGMP in this pathway. Alternatively, VEGF expression may be increased downstream of NO release, as reported before. ${ }^{18}$ The last possibility is that VEGF is under direct control of PDE5/cGMP and exerts its effects via eNOS/NO signalling (figure 1). This signalling pathway has been described by Park et al ${ }^{11}$ who demonstrated that sildenafil inhibits PDE5, which in turn leads to cGMP-activated HIF signalling. A third and final issue that requires further study is the effect of sildenafil on healthy pups. Russo et al demonstrate that in normal fetuses antenatal sildenafil administration decreases vascular branching. This may reflect a fine-tuned balance in cGMP activity. Although cGMP is required for cell survival and angiogenesis, sustained exposure of high cGMP levels also leads to cell death and apoptosis in lung endothelial cells, ${ }^{19}$ thus suggesting a defined window of cGMP activity in which it promotes pulmonary angiogenesis, whereas vascular growth is inhibited when cGMP is outside this window.

What are the next steps for further development of this treatment toward clinical application? Fetal and maternal toxicity are well studied by Russo et al, but postnatal toxicity and effects on long-term development remain inconclusive. Although rodent studies demonstrated that transplacental sildenafil administration does not affect rodent brain development and function, ${ }^{7}$ future studies are required to evaluate the effect of transplacental sildenafil on postnatal parameters of safety and efficacy.

In conclusion, these studies point towards VEGF/ NO-dependent vascular branching as a crucial step in the late development and maturation of the alveolocapillary unit. In adult animals, VEGF/NO signalling is necessary for maintenance of lung tissue architecture and regeneration. Sildenafil enhances expression of both VEGF and eNOS/cGMP and may thus be a strong stimulus for pulmonary angiogenesis and vasculogenesis. The dependence on VEGF for development and maintenance links developmental disorders to adult disease and may extend the results of the current study well beyond $\mathrm{CDH}$ alone.

Acknowledgements JA is funded by the Dutch Heart Foundation grant number $2014 T 064$.

Contributors JA designed the draft of the editorial, while HJB and AVN critically revised the manuscript.

Funding Hartstichting (grant number 2014 T064 (JA)).

Competing interests None declared.

Provenance and peer review Commissioned; externally peer reviewed.

\section{REFERENCES}

1 Russo FM, Toelen J, Eastwood MP, et al. Transplacental sildenafil rescues lung abnormalities in the rabbit model of diaphragmatic hernia. Thorax 2016;71:517-25.

2 Abman SH. Bronchopulmonary dysplasia: "a vascular hypothesis". Am J Respir Crit Care Med 2001;164(Pt 1):1755-6.

3 Voelkel NF, Vandivier RW, Tuder RM. Vascular endothelial growth factor in the lung. Am J Physiol Lung Cell Mol Physiol 2006;290:L209-21.

4 Warburton D, El-Hashash A, Carraro G, et al. Lung organogenesis. Curr Top Dev Biol 2010:90:73-158.

5 Healy AM, Morgenthau L, Zhu X, et al. VEGF is deposited in the subepithelial matrix at the leading edge of branching airways and stimulates neovascularization in the murine embryonic lung. Dev Dyn 2000;219:341-52.

6 van der Horst IW, Rajatapiti P, van der Voorn P, et al. Expression of hypoxia-inducible factors, regulators, and target genes in congenital diaphragmatic hernia patients. Pediatr Dev Pathol 2011;14:384-90.

7 Luong C, Rey-Perra J, Vadivel A, et al. Antenatal sildenafil treatment attenuates pulmonary hypertension in experimental congenital diaphragmatic hernia. Circulation 2011;123:2120-31.

8 Compernolle V, Brusselmans K, Acker T, et al. Loss of HIF-2alpha and inhibition of VEGF impair foetal lung maturation, whereas treatment with VEGF prevents fatal respiratory distress in premature mice. Nat Med 2002;8:702-10.

9 Jakkula M, Le Cras TD, Gebb S, et al. Inhibition of angiogenesis decreases alveolarization in the developing rat lung. Am J Physiol Lung Cell Mol Physiol 2000;279:L600-7.

10 Ladha F, Bonnet S, Eaton F, et al. Sildenafil improves alveolar growth and pulmonary hypertension in hyperoxia-induced lung injury. Am J Respir Crit Care Med 2005;172:750-6.

11 Park HS, Park JW, Kim HJ, et al. Sildenafil alleviates bronchopulmonary dysplasia in neonatal rats by activating the hypoxia-inducible factor signaling pathway. Am J Respir Cell Mol Biol 2013;48:105-13. 
12 Thébaud B, Ladha F, Michelakis ED, et al. Vascular endothelial growth factor gene therapy increases survival, promotes lung angiogenesis, and prevents alveolar damage in hyperoxia-induced lung injury: evidence that angiogenesis participates in alveolarization. Circulation 2005;112:2477-86.

13 Michaud SE, Dussault S, Groleau J, et al. Cigarette smoke exposure impairs VEGF-induced endothelial cell migration: role of NO and reactive oxygen species. J Mol Cell Cardiol 2006;41:275-84.

14 Weissmann N, Lobo B, Pichl A, et al. Stimulation of soluble guanylate cyclase prevents cigarette smoke-induced pulmonary hypertension and emphysema. Am J Respir Crit Care Med 2014;189:1359-73.

15 Kasahara Y, Tuder RM, Taraseviciene-Stewart L, et al. Inhibition of VEGF receptors causes lung cell apoptosis and emphysema. J Clin Invest 2000;106:1311-9.
16 Boucherat O, Morissette MC, Provencher S, et al. Bridging Lung Development with Chronic Obstructive Pulmonary Disease. Relevance of Developmental Pathways in Chronic Obstructive Pulmonary Disease Pathogenesis. Am J Respir Crit Care Med 2016;193:362-75.

17 Grover TR, Asikainen TM, Kinsella JP, et al. Hypoxia-inducible factors HIF-1alpha and HIF-2alpha are decreased in an experimental model of severe respiratory distress syndrome in preterm lambs. Am J Physiol Lung Cell Mol Physiol 2007;292:L1345-51.

18 Dulak J, Józkowicz A, Dembinska-Kiec A, et al. Nitric oxide induces the synthesis of vascular endothelial growth factor by rat vascular smooth muscle cells. Arterioscler Thromb Vasc Biol 2000;20:659-66.

19 Zhu B, Strada S, Stevens T. Cyclic GMP-specific phosphodiesterase 5 regulates growth and apoptosis in pulmonary endothelial cells. Am J Physiol Lung Cell Mol Physiol 2005;289:L196-206. 